\title{
BMJ Open Reducing antibiotic prescribing by enhancing communication of general practitioners with their immigrant patients: protocol for a randomised controlled trial (PARCA study)
}

\begin{abstract}
Dominique Lescure, ${ }^{1,2}$ Janneke van der Velden, ${ }^{3}$ Daan Nieboer, ${ }^{1}$ Wilbert van Oorschot, ${ }^{4}$ Rob Brouwer, ${ }^{5}$ Natascha Huijser van Reenen, ${ }^{3}$ Aimée Tjon-A-Tsien, ${ }^{2}$ Özcan Erdem, ${ }^{6}$ Margreet Vos, ${ }^{7}$ Alike van der Velden (1) , ${ }^{8}$ Jan Hendrik Richardus (D) , ${ }^{1,2}$ Hélène Voeten ${ }^{1,2}$
\end{abstract}

To cite: Lescure $D$, van der Velden J, Nieboer D, et al. Reducing antibiotic prescribing by enhancing communication of general practitioners with their immigrant patients: protocol for a randomised controlled trial (PARCA study). BMJ Open 2021;11:e054674. doi:10.1136/ bmjopen-2021-054674

- Prepublication history for this paper is available online. To view these files, please visit the journal online (http://dx.doi. org/10.1136/bmjopen-2021 054674).

Received 19 June 2021 Accepted 28 September 2021

Check for updates

(C) Author(s) (or their employer(s)) 2021. Re-use permitted under CC BY-NC. No commercial re-use. See rights and permissions. Published by BMJ.

For numbered affiliations see end of article.

Correspondence to

Dr Hélène Voeten;

hacm.voeten@rotterdam.nl

\section{ABSTRACT}

Introduction Although antibiotic use and antimicrobial resistance in the Netherlands is comparatively low, inappropriate prescription of antibiotics is substantial, mainly for respiratory tract infections (RTIs). General practitioners (GPs) experience pressure from patients with an immigration background to prescribe antibiotics and have difficulty communicating in a culturally sensitive way. Multifaceted interventions including communication skills training for GPs are shown to be most effective in reducing antibiotic prescription. The PARCA study aims to reduce the number of antibiotic prescriptions for RTIs through implementing a culturally sensitive communication intervention for GPs and evaluate it in a randomised controlled trial (RCT).

Methods and analysis A non-blinded RCT including 58 GPs (29 for each arm). The intervention consists of: (1) An E-learning with 4 modules of 10-15 min each; (2) A faceto-face training session in (intercultural) communication skills including role plays with a training actor and (3) Availability of informative patient-facing materials that use simple words (A2/B1 level) in multiple languages. The primary outcome measure is the number of dispensed antibiotic courses qualifying for RTIs in primary care, per 1000 registered patients. The secondary outcome measure is the number of all dispensed antibiotic courses, per 1000 registered patients. The intervention arm will receive the training in Autumn 2021, followed by an observation period of 6 winter months for which numbers of antibiotics will be collected for both trial arms. The GPs/practices in the control arm can attend the training after the observation period.

Ethics and dissemination The study protocol was approved by the Medical Ethics Review Committee of Erasmus MC, University Medical Center Rotterdam (MEC2020-0142). The results of the trial will be published in international peer-reviewed scientific journals and will be disseminated through national and international congresses. The project is funded by The Netherlands Organisation for Health Research and Development (ZonMw).

\section{Strengths and limitations of this study}

- This randomised controlled trial (RCT) in cities with large and diverse ethnic communities is unique in its focus on improving the communication of general practitioners (GPs) with their patients with an immigration background.

- Focus group discussions with immigrant patients and interviews with GPs and pharmacists are used to develop the content of a training intervention.

- The RCT includes a process evaluation with pre and post self-evaluations by GPs regarding their skills in cultural-sensitive communication, and skills to assess patient expectations and explain antibiotic non-prescribing to immigrant patients.

- Due to data restrictions, the outcome measures include antibiotics that are prescribed for all patients and not solely for immigrants.

- The primary outcome measure, that is, the number of dispensed antibiotic courses qualifying for respiratory tract infections in primary care, cannot be related to the precise medical indication.

Trial registration number NL9450.

\section{INTRODUCTION}

Most antibiotics are prescribed in primary care and mainly for respiratory tract infections (RTIs). ${ }^{12}$ Antibiotics for RTIs are often not prescribed according to the guidelines; they are prescribed when not indicated (overprescribing) and/or a non-first choice antibiotic is prescribed. Therefore, RTIs are worldwide the most frequent reasons for inappropriate antibiotic prescribing. ${ }^{34}$ In the Netherlands, a comparatively low-prescribing country, it was shown that nearly half $(46 \%)$ of all prescriptions for RTIs were not in accordance 
with the Dutch guidelines; overprescribing was highest for lower RTIs. ${ }^{5}$ Prescribing antibiotics appropriately can be challenging for general practitioners (GPs) and many factors interfere, like diagnostic uncertainty, risk-adverse behaviour, patients' expectations, perceived patient pressure and wanting to maintain a good doctor-patient relationship. ${ }^{56}$

An important factor related to prescribing behaviour is the GPs perception of patients' expectations; GPs generally perceive the expectation of patients to receive antibiotics as high. ${ }^{56}$ Moreover, GPs feel that patients try to exert pressure to be prescribed antibiotics. GPs, however, often seem to overestimate the patients' wish for antibiotics. McKay et al showed that the physician's perception of patients' desire for antibiotics was strongly associated with antibiotic prescribing, whereas the patients' actual desire for antibiotics was only modestly associated. ${ }^{7}$ Furthermore, it has been shown that patients' satisfaction is not primarily related to receiving antibiotics, but more to reassurance, adequate explanation and a physical examination. ${ }^{8}$

Prescribing antibiotics appropriately for persons with an immigration background can be especially challenging for GPs. Immigrants are often used to a high consumption of (easily accessible) antibiotics in their home country. They also have different cultural habits and often face a language barrier that could complicate the dialogue about proper use of antibiotics. ${ }^{9}$ This can make it more difficult to satisfy immigrants without prescribing antibiotics. Moreover, persons with an immigration background could have lower trust in GPs and possibly be less inclined to trust GPs' reasoning against the use of antibiotics. Perceived pressure to prescribe an antibiotic was shown to be particularly felt during consultations with patients with an immigration background..$^{11}$

Both the perceptions of the GPs and the expectations of immigrants have an influence on the interaction during consultation, in which the GP must decide whether to prescribe antibiotics. Although intercultural communication is challenging for doctors, it is sparsely trained for. Paternotte et al analysed videotaped consultations and showed that medical specialists in the Netherlands did practice some communication skills, such as listening and empathic communication behaviour, but specific intercultural communication skills, such as checking patients' expectations and language ability and being culturally aware, were not practised. ${ }^{12}$ In addition to the lack of training in intercultural communication among GPs, the interaction is further complicated by the fact that immigrants often have a low health literacy ${ }^{1314}$ and/or are low literate. $^{15}$

To improve the antibiotic prescription behaviour of GPs for their immigrant patients, training in (intercultural) communication skills is promising. In general, communication training for GPs has been found effective in reducing antibiotic prescriptions. ${ }^{16}{ }^{17}$ In addition, patient leaflets with information about common infections that are used during GP consultations are useful in reducing antibiotic prescriptions. ${ }^{18}$ Harmsen et al performed a randomised controlled trial (RCT) assessing the effectiveness of an intercultural communication training for Dutch GPs to decrease inequalities in care. They found an improvement in quality of care and in mutual understanding in consultations with immigrant patients. ${ }^{19}$

To date, we are not aware of any intervention for appropriate prescription of antibiotics by GPs for patients with an immigration background. There has been one small German study in which a leaflet for acute cough was adopted for Turkish immigrants. ${ }^{10}$ Reviews have shown that multifaceted interventions that include communication skills training for GPs are most effective for reducing antibiotic prescribing in general. ${ }^{20}{ }^{21}$ Specifically for GPs with an immigrant patient population, interventions should focus on increasing skills to efficiently explore concerns and expectations of immigrant patients, to reassure them and to provide understandable arguments to explain non-prescribing. ${ }^{52}$

The PARCA study (Reducing antibiotic prescribing by enhancing communication of general practioners with their immigrant patients) aims to reduce the number of antibiotic prescriptions for RTIs through implementing a culturally sensitive communication intervention for GPs and evaluate it in an RCT.

\section{METHODS AND ANALYSIS}

\section{Design and setting}

Non-blinded RCT in primary care with two, equally sized, parallel arms (intervention and control group) of GPs. GPs are randomised at individual level.

\section{Study sites and period}

The trial includes GPs working in the four largest cities in the Netherlands: Rotterdam, Amsterdam, The Hague and Utrecht (indicated as the G4). The G4 have the largest proportion of inhabitants with an immigration background (ie, born abroad or having at least one parent who was born abroad): $52 \%, 56 \%, 56 \%$ and $36 \%$, respectively. ${ }^{23}$ As our intervention aims to improve the communication between GPs and their immigrant patients, we focus specifically on GPs within the G4 with a practice in neighbourhoods with large numbers of immigrants $(>50 \%)$. Within each city, training sessions will be provided at central locations. The trial will be conducted from Autumn 2021 to Spring 2022.

\section{Study participants}

All GPs practising in the G4 who are interested to improve their communication with immigrant patients will be considered for enrolment. These GPs can subscribe individually (regardless of whether they work in a solo practice, duo practice or a group practice) or subscribe as a duo/group practice and participate with their fellow GPs from the same practice. In both cases, GPs will participate individually. The only inclusion criterion is that GPs use their own individual identification code (in Dutch: 
AGB-code) to prescribe antibiotics, which will allow us to extract data about antibiotics that are prescribed by individual GPs. The Dutch national healthcare system requires each GP to have such an individual identification code. While this is obligatory mainly because of health insurance payments, it enables us to link a GP to his/her individual prescribing behaviour.

We exclude all GPs who do not use an individual identification code. This refers to locums who often work at multiple practices and use the identification code of the registered GP, and to GPs who share an identification code with their fellow GP(s) within the same practice.

\section{Recruitment and reminders}

For recruitment we use professional networks, diverse social media channels, pitches in various online newsletters (eg, from the regional antibiotic resistance care networks), personal mailings sent by the Dutch Funds for GPs working in deprived neighbourhoods in the G4, and announcements during in-service training for GPs. In addition, GP practices in the G4 will be approached directly by phone. Mailings will be used to remind the GPs about the upcoming training and the data collection.

\section{Informed consent}

Participating GPs will receive information via email about the aim and content of the study. They will be ensured that participation is voluntary and that withdrawal from the study is possible at any time for any reason, without any consequences. Digital informed consent will be obtained prior to the start of the study.

\section{Randomisation, allocation concealment and blinding}

The participating GPs will be assigned to either the intervention or the control group through computer randomisation software. We will use stratified randomisation by stratifying the whole study population into two subgroups (GPs who subscribe individually and GPs who subscribe with colleagues from the same practice) and randomise either individuals (subgroup 1) or practice colleague groups (subgroup 2). In this way, GPs from the same practice will be allocated to the same study arm, thereby reducing the chance of contamination bias. The allocation ratio is $1: 1$.

The trial is not blinded as GPs will know whether they receive the intervention or not. The study investigators are also aware of the grouping because of the responsibility for inviting GPs to the training, for data collection and for statistical analysis.

\section{Intervention}

We will develop a tailored intervention for GPs, consisting of three components: An E-learning of four modules, a face-to-face training session and availability of simple informative patient-facing materials in multiple languages. The content of the intervention is based on existing courses of collaborating partner Pharos (the Dutch Expertise Centre on Health Disparities) and on the results of focus group discussions with various immigrant patient groups and interviews with GPs and pharmacists. The E-learning and the training session are accredited for continuing professional training.

The E-learning consists of four lessons of 10-15 min each. The main subjects will be: (1) cultural differences; (2) recognising low health literacy (among immigrants); (3) culturally sensitive communication about antibiotics and (4) learning how to communicate about antibiotics in a simple manner, and how to use the developed simple patient-facing materials as support during a consultation. The lessons will include relevant information materials and several movies, that is, of exemplary doctor-patient interactions during a consultation for RTIs, experiences of a GP with elaborated experience in communicating with immigrants, and experiences of an immigrant patient. The E-learning will contain questions to test the acquired knowledge of the GPs for accreditation purposes, and questions to explore problems experienced in daily practice which will be used during the face-to-face training session. The GPs can follow the E-learning modules at a convenient time within 2 weeks before the training session.

The training session will last 3 hours. It will consist of the following parts: (1) Learning and practising how to interact in a culturally sensitive way with immigrant patients, using specific intercultural skills; (2) Learning how to communicate with patients in simple words; (3) Learning and practising how to check whether the patient has understood the explanation using the teachback method and (4) Practising with the use of the developed patient materials to explain why antibiotics are not necessary. The newly learnt skills will be practised during role plays with a training actor. The participating GPs will also discuss video-taped consultations with intercultural patient-doctor interactions. An experienced trainer of Pharos, with a background as GP, will lead the training sessions. Beforehand the participants are offered a warm meal and during the training there will be coffee/tea breaks. Where possible, the training will be held at GP practices, to decrease the barrier for GPs to participate.

The third intervention component consists of simple informative patient-facing materials about antibiotics that can be used as support by GPs when they want to provide information about the correct use of antibiotics. First, we will develop a short animation movie that explains in simple images and words what antibiotics are, how they work, why they do not work in viral infections, how the immune system can fight infections itself, what antimicrobial resistance (AMR) is, and why GPs need to prescribe antibiotics prudently. The movie will be translated and subtitled and spoken (voice-over) in Arabic, Turkish, Tigrinya, Berber and English, which are common foreign languages in the Netherlands. The movie can be used by GPs on screens in their waiting rooms and during the consultation. Second, we will rewrite two existing texts about antibiotics to A2/B1 level to make them accessible for low-literate people. These texts are already available on the website of the Dutch College of General Practitioners 


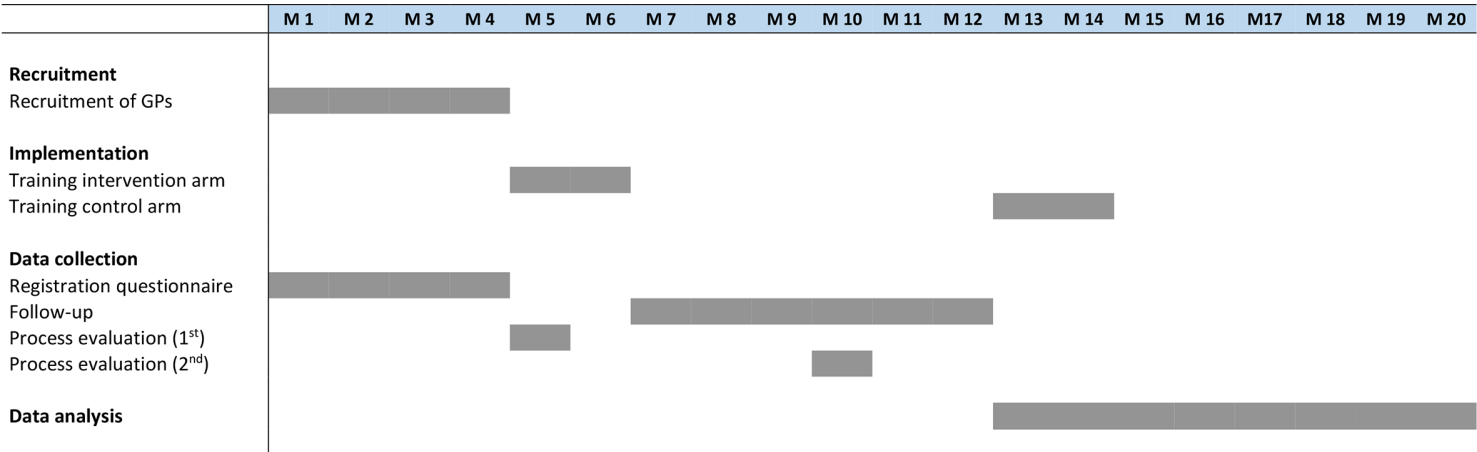

(www.thuisarts.nl) but are too difficult to read. The first text discusses why patients receive antibiotics or not, and the second text provides information about AMR. The texts will be translated in Arabic, Turkish, Tigrinya and English. GPs can use the simplified and translated information about antibiotics during their consultations to support their information provision to patients. Moreover, it is very easy to print this information so the patient can take it home.

The intervention will be implemented in the intervention arm in September/October 2021 and followed by an observation period of 6 winter months (November 2021-April 2022) during which numbers of dispensed antibiotics will be collected for both trial arms. The GPs/ practices in the control arm are given the opportunity to attend the training after the observation period (Spring 2022). Figure 1 presents the time schedule of the study.

\section{Patient and public involvement}

Patients and the public were not involved in this study, which focuses on GPs instead of patients.

\section{Data collection and management}

Data will be obtained from the Dutch Foundation of Pharmaceutical Statistics (SFK), which maintains an information system for pharmacists dispensing GP-prescribed drugs, including antibiotics. Of note is that in the SFK database the drugs dispensed are not linked to information on clinical indication. We, therefore, include antibiotics that qualify for RTIs (see below). Additionally, SFK only registers prescribed drugs that have actually been dispensed to patients. Arrangements will be made with SFK on how and when the data will be extracted and stored, and about ownership of the data. A similar agreement will be made with the GPs and pharmacists.

Data on individual background characteristics of each GP (eg, age, gender, years in practice) will be collected separately through an online registration questionnaire before the start of the intervention. Data extraction, storage and analysis follow standard safety procedures.

\section{Study outcomes}

Our primary outcome measure is the number of dispensed antibiotic courses qualifying for RTIs in primary care, per 1000 registered patients.
Registered patients refer to all patients who are registered with a specific GP. Due to formal data restrictions, we are unable to collect data on the country of origin of patients. As a result, the data will not exclusively include antibiotics that are dispensed to immigrants only but will also include antibiotics that are dispensed to other registered patients. To produce an outcome largely applicable to immigrant patients, we only include GPs working in neighbourhoods where most inhabitants $(>50 \%)$ are immigrants.

For eligible antibiotics, we selected all first and second choice antibiotics that qualify for RTIs in primary care according to the Dutch antibiotic guidelines and expert opinion. This produced the following selection of antibiotics: Doxycycline (J01AA02), Amoxicillin (J01CA04), Augmentin (J01CR02), Phenoxymethylpenicillin (J01CE02), Macrolides (J01FA) and Pheneticillin (J01CE05). With these selected antibiotics, we have included roughly two-thirds of the total amount of antibiotics prescribed within Dutch primary care. ${ }^{24} 25$

The secondary outcome measure is the number of dispensed courses of antibiotics for all infections (regardless of the indication), per 1000 registered patients.

All data for the primary and secondary outcomes will be retrieved retrospectively by SFK after the observation period. SFK will select oral antibiotics only and remove chronic/repeat prescriptions for the same antibiotic within two times the duration of the first prescription.

\section{Data handling}

All data will be anonymised by removing identifying personal information of the GPs and replacing it by a study number. The file containing the key between study numbers and identifying personal information of GPs will be password protected and kept in a separate folder only accessible to the main researcher. SFK will provide aggregated data regarding the number of dispensed antibiotic courses prescribed by participating GPs, without providing any individual patient information. These data will be linked to other data of the GPs (study arm, individual background characteristics, process evaluation outcomes) through their study number.

All study documents will be stored securely for at least 15 years at the Department of Public Health of Erasmus 
MC. A data management plan (DPM) is in place according to the requirements of the funding agency 'The Netherlands organisation for health research and development' (ZonMw). The second Excel-file will be used for analysis and reporting purposes and made available for verification and future research after all articles pertaining to the study have been published. Establishment of a data monitoring committee is not necessary because our intervention is not clinical and does not directly intervene on patients.

\section{Sample size}

With this multifaceted intervention we intend to demonstrate a statistically significant reduction in the rate of prescribed antibiotic courses qualifying for RTIs in primary care, from 150 to 125 antibiotic courses per 1000 registered patients, in a period of 6 winter months. This is a reduction of $16.6 \%$. Assuming the decrease of $16.6 \%$, a SD of 35 per 1000 registered patients, and a correlation of 0.40 between baseline and follow-up prescription rate, we would require 58 GPs to obtain $80 \%$ power at a significance level of 5\% (29 per RCT arm). Considering a drop-out of $15 \%$ we would require 66 GPs in total (33 in each arm).

The assumption on antibiotic prescription rate by GPs is derived from a previous study in the Netherlands, showing a prescription rate of 286 antibiotics/1000 registered patients/year. ${ }^{25}$ In our project, we only consider a selection of antibiotics, namely those that qualify for RTIs, which are roughly two thirds of all antibiotics; however, these antibiotics are mainly prescribed in the winter months (our follow-up period). Therefore, we assume 150 selected antibiotics $/ 1000$ registered patients $/ 6$ winter months. The assumed SD of 35 is derived from an unpublished working document of the Dutch SABEL (Spiegelinformatie Antibiotica Eerste Lijn) project on antibiotic prescription in general practice. (A.W. van der Velden, personal communication)

\section{Statistical analysis}

We will compare individual background characteristics and baseline measurements of the intervention and control group using data from the following 6-month periods: November 2019 to April 2020 (baseline option 1); November 2020 to April 2021 (baseline option 2) and November 2021 to April 2022 (follow-up). Due to COVID19 , we include two options for the baseline period. In the first year of the COVID-19 pandemic, it was shown that the number of antibiotic prescriptions in the Netherlands decreased significantly. ${ }^{26}$ We do not know whether the effect of the COVID-19 pandemic will continue in the coming years. Therefore, we have two baseline options. Because the control arm will not receive the intervention, it reflects the 'normal' situation regarding the number of prescribed antibiotics. If the primary outcome measure in the control arm during the follow-up is closer to baseline option 1, we will use this as baseline for the trial; if it is closer to baseline option 2, we will use this as baseline for the trial. Beside this primary analysis, we will use the other baseline option in a secondary (sensitivity) analysis.

To evaluate the effect of the intervention, we will perform a one-way analysis of covariance in which we statistically control for the number of dispensed antibiotics at baseline and the differences between the intervention and the control group. The number of antibiotics at baseline for both groups will be compared with the number of antibiotics during the 6 months following the intervention. We will perform our analysis primarily according to the intention-to-treat principle. In addition, we will include a per-protocol analysis in which the GPs in the intervention arm who did not participate in the training session, will be removed from the analysis. This allows us to examine the actual effect of the intervention.

If study participants discontinue participating in the trial, we will use their data that are collected until the point of discontinuation (eg, information about their gender, age, years in practice and the number of antibiotics).

\section{Missing values}

Missing data will not be replaced.

\section{Process evaluation}

Before the intervention, as well as 3 months after the intervention, participating GPs will be asked to fill in an online questionnaire to rate their own skills in culturalsensitive communication, skills to assess patient expectations, and skills to explain antibiotic non-prescribing to immigrant patients. In the second questionnaire, GPs in the intervention group will also be asked about their perceived relevance of the training, the applicability of the developed patient-facing materials in daily practice, and whether they feel the training improved their communication skills.

\section{Patient and public involvement}

This study is primarily among GPs and concerns their interaction with patients with a migratory background. For input into the study, GPs have been involved personally, through their professional organisations, and through focus group discussions. Focus group discussions have also been conducted with participants from various migratory backgrounds to obtain their views on the topic of the prescription of antibiotics by GPs. The results of the focus group discussions with both the professionals and the target population will be published as separate studies.

\section{ETHICS AND DISSEMINATION \\ Ethical considerations}

The need for formal ethical approval was waived by the Medical Ethics Review Committee of Erasmus MC, University Medical Center Rotterdam (MEC-2020-0142), after establishing that it does not fall under the Dutch Law on Medical Research. 


\section{Dissemination}

The research team will provide written personal feedback to all participating GPs at the end of the study through personal reports, in which their individual data regarding number of dispensed antibiotics will be mirrored to data of all participating GPs in the same RCT arm. Furthermore, the results of this trial will be reported in relevant academic journals and conferences. The methods and the results of the trial will be reported according to the Consolidated Standards of Reporting Trials (CONSORT) guidelines.

\section{DISCUSSION}

In Europe, many GPs struggle with providing adequate care to immigrants due to, among others, cultural issues, language difficulties and differences in expectations. ${ }^{2728}$ This can have a major impact when communication is needed about complex medical issues, such as antibiotics, in which GPs need to balance patient expectations, their own decision-making process, individual patient benefit and the community AMR problem. ${ }^{29}$ This paper describes the protocol of an RCT aiming to explore the effectiveness of a culturally sensitive communication training for GPs in reducing the number of prescribed antibiotics for RTIs. This is needed as the increased immigration brings about several intercultural communication challenges.

This study is unique in that it is situated in cities with large and diverse ethnic communities, and in its specific focus on improving the communication about antibiotics of GPs with their patients with an immigration background. Focus group discussions with various immigrant patients and interviews with GPs and pharmacists are used to develop the content of the training intervention. The study includes a process evaluation with pre-selfevaluations and post-self-evaluations by GPs regarding their skills in cultural-sensitive communication and skills to assess patient expectations and explain antibiotic nonprescribing to immigrant patients. On the other hand, we identify several limitations. Because of data restrictions, we cannot relate the prescribed antibiotics directly to the precise medical indication. This is mitigated by focusing on antibiotics that are used for RTIs in primary care. Data on the immigrant background of patients are not available, forcing us to include prescriptions for the complete patient population of a GP, without distinguishing between native and immigrant patients. By focusing on GPs in neighbourhoods where immigrants are the majority, we hope to minimise the dilution of our effect. Also, the outcome measure is the number of dispensed (and not originally prescribed) antibiotics, but we expect this to be of little influence because of high willingness of patients to take antibiotics. Another data limitation is that we only have access to the individual antibiotic prescription data of the participating GPs by using their personal identification codes. There is a risk that these identification codes are shared with non-participating colleagues in shared practices.
The emergence of the worldwide COVID-19 pandemic had a substantial influence on the design of our trial. The COVID-19 pandemic significantly reduced the number of antibiotic prescriptions in the Netherlands, probably because of changes in consultation behaviour. ${ }^{26}$ Also, the public health and social distancing measures have reduced the sentinel influenza virus positive detections. ${ }^{30}$ As a result, we decided to delay the implementation of our intervention by 1 year. The recruitment for this trial will be completed in Autumn 2021. The collection of primary data will conclude in Spring 2022. It is uncertain whether the COVID-19 pandemic will influence the willingness of GPs to participate in the trial and their antibiotic prescribing behaviour in the coming year. Still, we hope to succeed in performing the PARCA trial, which will allow us to make evidence-based recommendations to improve culturally sensitive communication skills of GPs in primary care.

\section{TRIAL STATUS}

This study is in the process of recruiting GPs, and it is expected to be completed in December 2022.

\section{Author affiliations}

${ }^{1}$ Department of Public Health, Erasmus MC, University Medical Center Rotterdam, Rotterdam, The Netherlands

${ }^{2}$ Department of Infectious Disease Control, Municipal Public Health Service Rotterdam-Rijnmond, Rotterdam, The Netherlands

${ }^{3}$ Pharos, Dutch Centre of Expertise on Health Disparities, Utrecht, The Netherlands

${ }^{4}$ Health Centre Zuidplein, Rotterdam, The Netherlands

${ }^{5}$ Health Centre Levinas, Pharmacy Ramleh, Rotterdam, The Netherlands ${ }^{6}$ Department of Research and Business Intelligence, Municipality of Rotterdam, Rotterdam, The Netherlands

${ }^{7}$ Department of Medical Microbiology and Infectious Diseases, Erasmus MC, University Medical Center Rotterdam, Rotterdam, The Netherlands

${ }^{8}$ Julius Center for Health Sciences and Primary Care, University Medical Center Utrecht, Utrecht, The Netherlands

Contributors Wv0, RB, AT-A-A, OE and MCV conceived the study, contributed to the protocol and are consulted yearly about the trial procedure. JvdV and NHVR conceived the study, contributed to the protocol and are actively involved in the development of the intervention. AWvdV conceived the study, contributed to the protocol, and are regularly asked for advice concerning the trial procedure. HV conceived of the study, initiated the study design, has written and composed the protocol, assists with the implementation and is the PhD cosupervisor of $\mathrm{DL}$. DN advised in the statistical analyses and performed the power calculation. JHR conceived of the study, has contributed extensively to the protocol and is the PhD supervisor of DL. DL is implementing the trial, has contributed extensively to the protocol, has written this manuscript, and will be conducting the primary statistical analysis. All authors contributed to refinement of the study protocol and approved the final manuscript.

Funding The work is supported by The Netherlands Organisation for Health Research and Development, ZonMw (Postbus 93245, 2509 AE Den Haag, The Netherlands), grant number 50-54100-98-209.

Disclaimer ZonMw had no role in the design of this study, and will not have any role during its execution, analyses, interpretation of the data, or decision to submit results.

\section{Competing interests None declared.}

Patient and public involvement Patients and/or the public were involved in the design, or conduct, or reporting, or dissemination plans of this research. Refer to the Methods section for further details.

Patient consent for publication Not applicable. 
Provenance and peer review Not commissioned; peer reviewed for ethical and funding approval prior to submission.

Open access This is an open access article distributed in accordance with the Creative Commons Attribution Non Commercial (CC BY-NC 4.0) license, which permits others to distribute, remix, adapt, build upon this work non-commercially, and license their derivative works on different terms, provided the original work is properly cited, appropriate credit is given, any changes made indicated, and the use is non-commercial. See: http://creativecommons.org/licenses/by-nc/4.0/.

\section{ORCID iDs}

Alike van der Velden http://orcid.org/0000-0002-9443-2837

Jan Hendrik Richardus http://orcid.org/0000-0003-0564-6313

\section{REFERENCES}

1 Petersen I, Hayward AC, Subgroup SS, SACAR Surveillance Subgroup. Antibacterial prescribing in primary care. J Antimicrob Chemother 2007;60 Suppl 1:i43-7.

2 van den Broek d'Obrenan J, Verheij TJM, Numans ME, et al. Antibiotic use in Dutch primary care: relation between diagnosis, consultation and treatment. J Antimicrob Chemother 2014;69:1701-7.

3 Costelloe C, Metcalfe C, Lovering A, et al. Effect of antibiotic prescribing in primary care on antimicrobial resistance in individual patients: systematic review and meta-analysis. BMJ 2010;340:c2096.

4 Butler CC, Hillier S, Roberts Z, et al. Antibiotic-resistant infections in primary care are symptomatic for longer and increase workload: outcomes for patients with E. coli UTIs. Br J Gen Pract 2006;56:686-92.

5 Dekker ARJ, Verheij TJM, van der Velden AW. Inappropriate antibiotic prescription for respiratory tract indications: most prominent in adult patients. Fam Pract 2015;32:401-7.

6 Akkerman AE, Kuyvenhoven MM, van der Wouden JC, et al. Determinants of antibiotic overprescribing in respiratory tract infections in general practice. J Antimicrob Chemother 2005;56:930-6.

7 McKay R, Mah A, Law MR, et al. Systematic review of factors associated with antibiotic prescribing for respiratory tract infections. Antimicrob Agents Chemother 2016;60:4106-18.

8 Welschen I, Kuyvenhoven MM, Hoes AW, et al. Effectiveness of a multiple intervention to reduce antibiotic prescribing for respiratory tract symptoms in primary care: randomised controlled trial. BMJ 2004;329:431.

9 Norris P, Ng LF, Kershaw V, et al. Knowledge and reported use of antibiotics amongst immigrant ethnic groups in New Zealand. $J$ Immigr Minor Health 2010;12:107-12.

10 Sahlan S, Wollny A, Brockmann S, et al. Reducing unnecessary prescriptions of antibiotics for acute cough: adaptation of a leaflet aimed at Turkish immigrants in Germany. BMC Fam Pract 2008;9:57.

11 Hogenhuis CC, Grigoryan L, Numans MM, et al. Differences in antibiotic treatment and utilization of diagnostic tests in Dutch primary care between natives and non-western immigrants. Eur J Gen Pract 2010;16:143-7.

12 Paternotte E, Scheele F, Seeleman CM, et al. Intercultural doctor-patient communication in daily outpatient care: relevant communication skills. Perspect Med Educ 2016;5:268-75.

13 Kreps GL, Sparks L. Meeting the health literacy needs of immigrant populations. Patient Educ Couns 2008;71:328-32.
14 Wångdahl J, Lytsy P, Mårtensson L, et al. Poor health and refraining from seeking healthcare are associated with comprehensive health literacy among refugees: a Swedish cross-sectional study. Int J Public Health 2018;63:409-19.

15 Buisman M, Houtkoop W. Laaggeletterdheid in kaart, 2014. Available: https://www.lezenenschrijven.nl/wat-doen-wij/oplossingvoor-je-vraagstuk/laaggeletterdheid-kaart [Accessed 20 May 2021]

16 Cals JWL, Butler CC, Hopstaken RM, et al. Effect of point of care testing for $\mathrm{C}$ reactive protein and training in communication skills on antibiotic use in lower respiratory tract infections: cluster randomised trial. BMJ 2009;338:b1374.

17 Strumann C, Steinhaeuser J, Emcke T, et al. Communication training and the prescribing pattern of antibiotic prescription in primary health care. PLoS One 2020;15:e0233345.

18 de Bont EGPM, Alink M, Falkenberg FCJ, et al. Patient information leaflets to reduce antibiotic use and reconsultation rates in general practice: a systematic review. BMJ Open 2015;5:e007612.

19 Harmsen H, Bernsen R, Meeuwesen L, et al. The effect of educational intervention on intercultural communication: results of a randomised controlled trial. Br J Gen Pract 2005;55:343-50.

20 Dyar OJ, Beović B, Vlahović-Palčevski V, et al. How can we improve antibiotic prescribing in primary care? Expert Rev Anti Infect Ther 2016;14:403-13.

21 van der Velden AW, Pijpers EJ, Kuyvenhoven MM, et al. Effectiveness of physician-targeted interventions to improve antibiotic use for respiratory tract infections. Br J Gen Pract 2012;62:e801-7.

22 Mangione-Smith R, Elliott MN, Stivers T, et al. Racial/ethnic variation in parent expectations for antibiotics: implications for public health campaigns. Pediatrics 2004;113:e385-94.

23 CBS. Hoeveel mensen met een migratieachtergrond wonen in Nederland? 2021. Available: https://www.cbs.nl/nl-nl/dossier/ dossier-asiel-migratie-en-integratie/hoeveel-mensen-met-eenmigratieachtergrond-wonen-in-nederland- [Accessed 14 Jun 2021].

24 NIVEL. Percentage patiënten dat antibioticum krijgt voorgeschreven daalt iets, 2018. Available: https://www.nivel.nl/nl/nieuws/ percentage-patienten-dat-antibioticum-krijgt-voorgeschreven-daaltiets [Accessed 14 Jun 2021].

25 van der Velden AW, Kuyvenhoven MM, Verheij TJM. Improving antibiotic prescribing quality by an intervention embedded in the primary care practice accreditation: the ARTI4 randomized trial. $J$ Antimicrob Chemother 2016;71:257-63.

26 van de Pol AC, Boeijen JA, Venekamp RP, et al. Impact of the COVID-19 pandemic on antibiotic prescribing for common infections in the Netherlands: a primary care-based observational cohort study. Antibiotics 2021;10. doi:10.3390/antibiotics10020196. [Epub ahead of print: 18 Feb 2021].

27 Goth U, Berg J, Akman H. The Intercultural challenges of general practitioners in Norway with migrant patients. Int J Migr Health Soc Care 2010;6:26-33.

28 Jensen NK, Norredam M, Draebel T, et al. Providing medical care for undocumented migrants in Denmark: what are the challenges for health professionals? BMC Health Serv Res 2011;11:154.

29 van der Zande MM, Dembinsky M, Aresi G, et al. General practitioners' accounts of negotiating antibiotic prescribing decisions with patients: a qualitative study on what influences antibiotic prescribing in low, medium and high prescribing practices. BMC Fam Pract 2019;20:172.

30 Adlhoch C, Mook P, Lamb F, et al. Very little influenza in the WHO European Region during the 2020/21 season, weeks 402020 to 8 2021. Euro Surveill 2021;26. 